\title{
Resistencia a los antihelmínticos en bovinos del nordeste de Corrientes (Argentina)
}

\author{
Lobayan, S.I. ${ }^{1}$; Schapiro, J.H. ${ }^{2}$; Fiel, C.A. ${ }^{3}$; Zabalo, M.M. ${ }^{4}$; Roselli, J.G. ${ }^{4}$ \\ ${ }^{1}$ Esc.Veterinaria, Univ.Salvador (USAL), Ruta Nac.14, Km 728, Virasoro (Corrientes, Argentina). \\ ${ }^{2}$ Esc.Veterinaria (USAL, Pilar) y Área Parasit.INTA Castelar. ${ }^{3}$ Univ.Nac.Centro Prov.Bs.As. (UNCPBA). \\ ${ }^{4}$ Alumnos practicantes USAL. E-mail: sergio.lobayan@usal.edu.ar
}

\begin{abstract}
Resumen
Lobayan, S.I.; Schapiro, J.H.; Fiel, C.A.; Zabalo, M.M.; Roselli, J.G.: Resistencia a los antihelmínticos en bovinos del nordeste de Corrientes (Argentina). Rev. vet. 28: 2, 138-140, 2017. El objetivo del trabajo fue determinar la prevalencia de la resistencia a los antiparasitarios contra los nematodes gastrointestinales de bovinos en el nordeste de Corrientes. El área de estudio comprendió 4 establecimientos ganaderos de los departamentos Ituzaingó y Santo Tomé. El periodo de estudio abarcó de marzo de 2014 a agosto de 2016. La unidad de análisis fue el ternero destete. El método utilizado fue el test de reducción del conteo de huevos. La prevalencia de la resistencia antihelmíntica a la ivermectina en los 4 establecimientos ganaderos muestreados en este estudio fue del $100 \%$ y para el benzimidazol fue del $25 \%$. Los géneros parasitarios resistentes a la ivermectina fueron Haemonchus y Cooperia. Estos resultados demuestran un aumento de la resistencia antihelmíntica si se comparan con estudios previos realizados en otros lugares del país.
\end{abstract}

Palabras clave: bovino, nematodes gastrointestinales, resistencia a antihelmínticos.

\begin{abstract}
Lobayan, S.I.; Schapiro, J.H.; Fiel, C.A.; Zabalo, M.M.; Roselli, J.G.: Resistance to antiparasitics in cattle from northeast of Corrientes (Argentina). Rev. vet. 28: 2, 138-140, 2017. The objective of this research was to determine the prevalence of the resistance to antiparasitics of bovine gastrointestinal nematodes in the northeast of Corrientes. The studied area comprised 4 livestock establishments in Ituzaingó and Santo Tomé districts. The study period ran from March 2014 to August 2016. The analysis unit was the weaning calf. The method used was the faecal egg count reduction test. The prevalence of the anthelmintic resistance to ivermectin in the four livestock establishments sampled in this study was of $100 \%$ and $25 \%$ to benzimidazole. The parasite genders resistant to ivermectina were Haemonchus and Cooperia. These results demonstrate an increase in anthelmintic resistance when compared with previous studies elsewhere in the country.
\end{abstract}

Key words: cattle, gastrointestinal nematodes, antihelmintic resistance.

\section{INTRODUCCIÓN}

En los sistemas de producción ganadera, la problemática de las parasitosis ocasiona importantes pérdidas económicas y hace que su control sea un condicionante de la competitividad de los sistemas. Debido a la eficacia de los antihelmínticos disponibles en el mercado y a la posibilidad de utilizar otras metodologías de control, los productores basan toda su estrategia de prevención en un uso indiscriminado y continuo del recurso químico ${ }^{5}$.

Diagnóstico y control son dos acciones inseparables de cualquier programa sanitario. En este caso no solamente basta conocer el agente causal, sino también es imprescindible determinar en forma precoz el grado

Recibido: 6 diciembre 2016 / Aceptado: 28 abril 2017 de sensibilidad de las poblaciones parasitarias frente a los grupos químicos disponibles ${ }^{6}$.

En oportunidad de llevarse a cabo el Proyecto de Cooperación Técnica de la FAO (PCT/RLA/2364) "Resistencia antihelmíntica en parásitos gastrointestinales de ovinos", desarrollado en 1994-1995, el 43\% de los establecimientos muestreados en Argentina tenía nematodes gastrointestinales resistentes. Los resultados mostraron que sólo el $38 \%$ de los rodeos ovinos estaban parasitados por nematodes susceptibles, en tanto que en bovinos, la resistencia estaba presente en el $60 \%$ de los rodeos estudiados y la mayoría de ellos (55\%) era resistente al grupo ivermectina ${ }^{1}$.

Así, la resistencia antihelmíntica fue catalogada como un problema que debía ser considerado por las autoridades de sanidad animal, la industria y los sectores de la producción. Dado que para Argentina la 
producción de carne bovina es la principal actividad ganadera, los resultados del estudio de prevalencia de la resistencia antihelmíntica en ovinos solo tuvieron impacto sobre una porción de los productores y de los profesionales ligados a la cría de ovinos que estaban en condiciones de entender el problema ${ }^{1}$.

Para endo y ectoparásitos la resistencia a las drogas ha sido definida con algunas variantes, en forma consistente con las definiciones propuestas por el Comité de Expertos en Insecticidas de la Organización Mundial de la Salud (OMS) en 1957 y el Grupo de Expertos sobre Resistencia de la FAO en el año 1967.

En esta comunicación se utiliza como definición de resistencia a "la detección por medio de pruebas sensitivas, de un aumento significativo de individuos dentro de una misma especie y población de parásitos capaces de tolerar dosis de drogas que han probado ser letales para la mayoría de individuos de la misma especie". Sobre esta base es posible no solo determinar cuál es la especie de nematodo mayormente involucrada sino también su grado de sensibilidad a los compuestos comerciales disponibles para su control ${ }^{7}$.

El objetivo del presente trabajo fue determinar la prevalencia de la resistencia antihelmíntica de los nematodes gastrointestinales de bovinos en el nordeste de la Provincia de Corrientes (Argentina).

\section{MATERIAL Y MÉTODOS}

Hasta el presente el método in vivo más utilizado en todo el mundo para detectar la resistencia de los nematodes es el test de reducción del conteo de huevos $(\mathrm{TRCH})^{3}$, el cual compara los valores promedios del recuento de huevos por gramo de materia fecal (HPG) antes y después del tratamiento ${ }^{9}$.

La selección de los rodeos se hizo por un muestreo de conveniencia, teniendo en cuenta la disponibilidad de los terneros, la distancia entre los campos y el laboratorio y la predisposición de los propietarios a participar en el estudio. El área de estudio incluyó cuatro establecimientos ganaderos de la Provincia de Corrientes, tres en el Departamento Ituzaingó y uno en el Departamento Santo Tomé. El periodo de estudio abarcó de marzo de 2014 a agosto de 2016.

La unidad de análisis fue el ternero destete. Se realizó la extracción de materia fecal del recto para efectuar los estudios coprológicos: HPG según técnica McMaster modificada por Roberts y O'Sullivan (1949) ${ }^{10}$, y coprocultivo por el método Corticelli y Lai.(1963) ${ }^{4}$ para la identificación y cuantificación del género de las larvas sobrevivientes al tratamiento por medio de las claves de Niec (1968) ${ }^{8}$.

Como criterio de inclusión se utilizó el rango de edad comprendido entre 6 y 12 meses; también que los terneros no hayan recibido tratamiento antiparasitario 60 días previos a la realización de la prueba y que en el análisis coprológico cuantitativo tuvieran un HPG superior a 100.
Se evaluaron los tres grupos (drogas) de antiparasitarios utilizados en la región: el levamisol, los benzimidazoles y las ivermectinas (variables independientes). La variable dependiente (cuantitativa discreta) fue la determinación de HPG. El trabajo en cada establecimiento contempló jornadas de campo y de laboratorio según el siguiente esquema:

1. Primera jornada de campo: se pesaron los terneros, se extrajeron las muestras de materia fecal y se las identificó con el número de caravana correspondiente a cada unidad experimental.

2. Primera jornada de laboratorio: se realizó el recuento de HPG de las muestras y se consideraron aquéllas cuyos valores fueran superiores a 100. Los resultados obtenidos se cargaron en archivos Excel, quedando armados 4 grupos homogéneos en cuanto al valor de $\mathrm{HPG}^{1}$.

3. Segunda jornada de campo: se aplicaron los tratamientos a cada grupo de terneros, considerándose esta fecha como día 0 de la experiencia.

4. Tercera jornada de campo: 14 días después se extrajeron muestras de materia fecal de los 4 grupos experimentales.

5. Segunda jornada de laboratorio: nuevamente se efectuó el recuento HPG de cada animal, y se realizó cultivo de larvas en cada grupo experimental (pool), calculándose previamente el porcentaje de reducción del conteo de huevos.

El porcentaje de eficacia del TRCH se calculó mediante el método de la World Association for the Advancement of Veterinary Parasitology (WAAVP) ${ }^{5}$, multiplicando $100 x(1-[\mathrm{T} 2 / \mathrm{C} 2])$, donde $\mathrm{T} 2$ es el promedio de HPG del grupo tratado el día 14 , y C2 es el promedio de HPG de los animales no tratados en el día $14^{5}$. Para que los resultados sean considerados estadísticamente válidos, se estableció que cada grupo debía estar formado con un mínimo de diez animales.

Según el método utilizado, la presencia de resistencia antihelmíntica se asocia con un TRCH por debajo del $90 \%$, y cuando el límite inferior del intervalo de confianza para el $95 \%$ sea menor al $90 \%{ }^{3}$.

6. Tercera jornada de laboratorio: 10 días después se realizó la identificación de los géneros parasitarios de los coprocultivos y en base al número de larvas hallado se calculó el porcentaje de cada género.

\section{RESULTADOS}

1. En las primeras jornadas de campo, en los 4 establecimientos estudiados se extrajeron muestras de materia fecal a 295 terneros.

2. En las primeras jornadas de laboratorio se conformaron los grupos con 180 terneros con HPG superior a 100 .

3. En las segundas jornadas de campo se hicieron las aplicaciones de los antiparasitarios a los cuatro grupos.

4. En las terceras jornadas de campo se extrajeron las muestras de materia fecal de los cuatro grupos. 
Tabla 1. Eficacia de cada droga antiparasitaria (en \%), según el establecimiento (E).

\begin{tabular}{cccc}
\hline E & ivermectina & benzimidazoles & levamisol \\
\hline 1 & 34,0 & 94,0 & 100 \\
2 & 21,2 & 91,2 & 96,2 \\
3 & 34,9 & 91,8 & 99,9 \\
4 & 37,2 & 72,4 & 99,3 \\
\hline
\end{tabular}

Tabla 2. Géneros (\%) en los coprocultivos de los grupos testigos en cada establecimiento (E).

\begin{tabular}{ccccc}
\hline E & Trichostrongylus & Haemonchus & Cooperia & Oesophagostomun \\
\hline 1 & 6 & 50 & 35 & 9 \\
2 & 13 & 35 & 22 & 30 \\
3 & 30 & 20 & 28 & 22 \\
4 & 22 & 36 & 30 & 12 \\
\hline
\end{tabular}

Tabla 3. Porcentaje de géneros parasitarios sobrevivientes a la ivermectina (en \%), por establecimiento (E).

\begin{tabular}{ccc}
\hline E & Haemonchus & Cooperia \\
\hline 1 & 52 & 48 \\
2 & 64 & 36 \\
3 & 32 & 68 \\
4 & 70 & 30 \\
\hline
\end{tabular}

5. Se determinó la cantidad de HPG de los 4 grupos de los 4 establecimientos. Se cargaron los datos en las planillas para el cálculo del TRCH para cada antiparasitario. Los resultados se observan en la Tabla 1.

6. En las terceras jornadas de laboratorio se realizaron las identificaciones de los géneros parasitarios de los coprocultivos de los cuatro grupos testigo, cuyos resultados se consignan en la Tabla 2.

En la Tabla 3 se observan los géneros sobrevivientes a los tratamientos con ivermectina de los 4 grupos. Los cultivos de bencimidazoles y levamisol fueron negativos.

\section{DISCUSIÓN}

Se puede afirmar que la prevalencia de la resistencia antihelmíntica en los 4 establecimientos ganaderos muestreados en este estudio fue del $100 \%$ para la ivermectina y del $25 \%$ para los benzimidazoles. La resistencia a la primera de ellas abarcó primariamente al género Haemonchus sp y secundariamente a Cooperia sp. Tales resultados demuestran un aumento de la resistencia al compararlos con relevamientos anteriores, donde fue del $55 \%$ para ivermectina y del $10 \%$ para benzimidazoles ${ }^{1}$.
Lo antedicho se ve ratificado por el estudio realizado por la Red Interinstitucional de Investigación y Experimentación en Enfermedades Parasitarias (RIEP) durante los años 2014 y 2015, donde sobre 62 establecimientos se detectó resistencia en el $95,2 \%$ de ellos, con resistencia a ivermectina en el $93,5 \%$, a ricobendazole en el 27,9\% y resistencia múltiple (a ambos) en el 26,2\% (artículo en prensa). En dicho estudio la resistencia a IVM abarcó primariamente al género Cooperia sp y secundariamente a Haemonchus sp.

De la totalidad de establecimientos analizados por el TRCH, diez de ellos se ubicaron en la Provincia de Corrientes, donde todos presentaron resistencia a ivermectina, uno de ellos resistencia a febendazol, dos con resistencia al ricobendazole, dos con resistencia múltiple, y todos fueron susceptibles al levamisol ${ }^{2}$.

\section{REFERENCIAS}

1. Caracostantógolo JL et al. 2006. Evaluación de la resistencia a los antihelmínticos en rumiantes en Argentina. Serie Producción y Sanidad Animal (FAO), p. 7-14.

2. Cetrá B. 2016. Resistencia antihelmíntica en bovinos en la Provincia de Corrientes, Argentina. Noticias y Comentarios, EEA INTA Mercedes, $\mathrm{N}^{\circ} 534$.

3. Coles GC et al. 1992. Methods for the detection of antihelmintic resistance in nematodos of veterinary importance. Vet Parasitol 44: 35-44.

4. Corticelli B, Lai M. 1963. Studies on the technique of culture of infective larvae of gastrointestinal strongyles of cattle. Acta Med Vet Napoli 9: 347-357.

5. Cristel SL, Suarez VH. 2006. Resistencia antihelmíntica: evaluación de la prueba de reducción del conteo de huevos. Rev Investig Agropec (RIA) 35: 29-43.

6. Nari A, Henrioud AN. 1987. Enfoque epidemiológico sobre el diagnóstico y control de resistencia a antihelmínticos en ovinos, Ed. Hemisferio Sur, Montevideo, p. 60.

7. Nari A. 2001. Diagnóstico y control de resistencia antihelmíntica en pequeños rumiantes. Anales II Congreso Latinoamericano de Especialistas en Pequeños Rumiantes y Camélidos Sudamericanos, Mérida, Yucatán, México.

8. Niec R. 1968. Cultivo e identificación de larvas infectantes de nematodes gastrointestinales del bovino y ovino. INTA, Manual Técnico $N^{\circ}$, p. 1-37

9. Presidente PJ, Anderson N, Waller PJ. 1985. Methods for detection of resistance to anthelmintics. En: Resistance in nematodes to anthelmintic drugs (Anderson $\mathrm{N}$, Waller PJ Ed.), CSIRO, Division of Animal Health, Canberra, Australia, p. 13-27.

10. Roberts FH, O'Sullivan PJ. 1949. Methods for egg counts and larvae cultures for strongyles infesting the gastro-intestinal tract of cattle. Aust J Agr Res 1: 99-102. 